\title{
The Agreement Rate about Unintended Pregnancy and Its Relationship with Postpartum Depression in Parents of Preterm and Term Infants
}

\author{
Maryam Ghorbani' ${ }^{1}$, Mahrokh Dolatian ${ }^{2 *}$, Jamal Shams³ ${ }^{3}$, Hamid Alavi-Majd ${ }^{4}$ \\ ${ }^{1}$ Shahid Beheshti University of Medical Sciences, Tehran, Iran \\ ${ }^{2}$ Department of Reproductive Health and Midwifery, School of Nursing and Midwifery, Shahid Beheshti \\ University of Medical Sciences, Tehran, Iran \\ ${ }^{3}$ Department of Psychiatry, Behavioral Research Center, Shahid Beheshti University of Medical Sciences, \\ Tehran, Iran \\ ${ }^{4}$ Department of Biostatistics, School of Paramedical Sciences, Shahid Beheshti University of Medical Sciences, \\ Tehran, Iran \\ Email: mhdolatian@gmail.com
}

Received 6 August 2015; accepted 22 September 2015; published 25 September 2015

Copyright (C) 2015 by authors and Scientific Research Publishing Inc.

This work is licensed under the Creative Commons Attribution International License (CC BY).

http://creativecommons.org/licenses/by/4.0/

C) (i) Open Access

\begin{abstract}
Introduction: Postpartum depression (PPD) affects women's health and self-confidence, and infant's social, emotional, cognitive and even physical development. Studies show that parents of preterm infants frequently experience symptoms of depression and anxiety. Women with unintended pregnancy are subjected to more risk of depression than women with planned pregnancy. Unintended pregnancy may lead to increased maternal exposure to psychosocial stressors, reduced social support by the spouse, increased levels of depressive symptoms and decreased life satisfaction. Findings: No significant difference was observed between term and preterm infants' mothers $(p=0.85)$ in terms of postpartum depression. However, two groups of fathers in terms of depression showed a significant difference $(p=0.045)$. McNemar's test showed that parents of term infants $(K=0.322, p=0.077)$, and parents of preterm infants $(k=0.17, p=0.144)$ agreed with each other on unintended pregnancy. Conclusion: Fathers of preterm infants are at higher risk for mental disorders than fathers of term infants and they need more attention in future studies.
\end{abstract}

"Corresponding author.

How to cite this paper: Ghorbani, M., Dolatian, M., Shams, J. and Alavi-Majd, H. (2015) The Agreement Rate about Unintended Pregnancy and Its Relationship with Postpartum Depression in Parents of Preterm and Term Infants. Open Journal of Nursing, 5, 797-804. http://dx.doi.org/10.4236/ojn.2015.59084 


\section{Keywords}

\section{Prematurity, Parents, Depression, Postpartum Depression, Unintended Pregnancy}

\section{Introduction}

For most women, pregnancy is a time of positive changes, but it can also be a time of physical and psychological challenges, as pregnancy is associated with hormonal changes and can therefore increase susceptibility to incidence or recurrence of depression [1]. Postpartum depression is effective not only on women's health and selfconfidence as a mother, but also on infant's social, emotional, cognitive and even physical development. Postpartum depression has been reported 10\% - 15\% in developed countries and 16\% to 36\% in developing countries. Numerous risk factors associated with postpartum depression have been recognized including mother's major depressive disorder, depression during pregnancy and family history of depression. Other important factors in some low-income countries include family fragmentation, lack of social support, dissatisfaction with having a baby girl, no breastfeeding, stressful life events, domestic violence, HIV/AIDS, infant diseases, infant prematurity and unintended pregnancy [1] [2].

Since changes occur in women's psychological and physiological function in the postpartum period, preterm delivery is likely to increase maternal distress and slow her physical recovery because of the special care required by a preterm infant [3]. The prevalence of preterm birth has been reported $5 \%$ in developed countries and $25 \%$ in developing countries. In Iran, the prevalence of preterm birth has been reported between $5.6 \%$ and $13.4 \%$ [4]. Although advances in technology result in more survival of preterm infants, mortality still remains high and imposes more emotional and financial burden to both family and the healthcare system [5].

Potentially stressful factors that can affect parents of preterm infants include problems related to the infant's health at the hospital and after discharge from the hospital, reduction in accountability, and concerns about the infant's mental development. Postpartum depression is a common experience in preterm infants' mothers [6]. According to several studies, experiencing emotional distress in parents of preterm infants is high and high levels of depression and anxiety symptoms appear during their baby's stay in the NICU [5].

Few studies have also investigated the effects of unintended pregnancy and have shown that women with an unintended pregnancy are at increased risk for depression compared to women with planned pregnancy. Unintended pregnancy may lead to increased maternal exposure to psychosocial stressors, reduced social support by the spouse, increased levels of depressive symptoms and decreased life satisfaction [1].

The aim of this study was to compare postpartum depression in parents of preterm and term infants, and review their agreement rate about unwanted pregnancy and its relationship with postpartum depression.

\section{Patients and Methods}

The current study used a descriptive-comparative, analytical design. The study population comprised parents of all preterm and term infants who presented at health centers in Qom, Iran to receive postpartum cares or to have their infants vaccinated. According to statistical analyses, the sample size was calculated as 82 couples and the subjects were selected using multistage stratified sampling during December 2012 to March 2013. Therefore, Because of existing different economic statue in Qom, the city was first divided into four economic classes. (The first stage of sampling was cluster sampling). All available health centers in the city were then listed and the sample size was distributed among the centers proportional to the number of their patients. (The second stage of sampling was quota sampling) Finally, centers from each class were randomly selected. Individuals were only recruited if they did not have any mental illnesses or a history of such diseases, were not on antipsychotic medicines such as antidepressants, and had no history of narcotic or psychoactive drug abuse. Parents of infants with any congenital or neurological defects were not included.

The present study used the following questionnaires:

Part one:

A questionnaire for the fathers:

The questionnaire consisted of two parts. The first part covered questions on demographic characteristics, having the intention to have a baby, the desired infant gender, and the history of mental illnesses of the first-degree 
relatives (6 items). The second part involved the adverse events which occurred to the father during his wife's pregnancy (27 items).

A questionnaire for the mothers:

The questionnaire included four parts. The first 12 items dealt with demographic characteristics of the mother, socioeconomic status of the family. The second part covered information on previous and current pregnancy (38 items). The third part of the questionnaire involved infant gender and their information and problem occurred because of infants after birth (15 items). The last 27 items explored the adverse pregnancy-related events.

The validity of these two questionnaires (demographic and obstetric) was determined with content validity. Moreover, in order to confirm the test-retest reliability of the questionnaire, 10 eligible couples who visited the mentioned health centers were selected and asked to complete the questionnaire twice with a two-week interval. The internal consistency of the tool was then computed as $98 \%$.

\section{Part two:}

Beck Depression Inventory: To measure fathers' depression in this study, the-second edition of depression inventory was used. This is a self-report questionnaire with 21 items, to assess depression in adults and adolescents over 13 years and is scored Likertstyle according to the severity of symptoms. Among these items, 2items are dedicated to emotion, 11 items to cognition, 2 items to apparent behaviors, 5 items to somatic symptoms, and 1 item to interpersonal semiotics. Thus this scale determines different degrees of depression from mild to very severe and the subjects were divided into four groups considering the sum of the scores obtained: minimal or no depression (0 - 13 score), indicates mild depression(14 - 19 score),moderate depression (20 - 28 score)and severe depression (29 - 63 score) [7].

Edinburgh Postpartum Depression Scale: To assess mothers' depression status in this study, the globally applied screening scale for postpartum depression, Edinburgh depression scale, was used. The questionnaire includes 10 items about the common symptoms of depression based on Likertstyle. Mothers select the responses which show how they felt more often in the last week. Each item has four options and a score of $0-3$. The sum of total score is $0-30$ [8]. A score above 13 in the postpartum period was considered as depressed and a score lower than 13 as non-depressed.

In several studies, Cronbach's alpha coefficient of the questionnaire was reported 0.83 to 0.9 with high content validity [5]. To determine the validity and reliability of the questionnaire in Iranian population, and to determine the depression degree and estimate the effects of clinical trial, Dobson et al. in their descriptive-applied study in 2007 titled "Psychometric characteristics of the Beck Depression Inventory-II in a large sample of patients with major depressive disorder" evaluated 354 individuals who were diagnosed with major depression and were relatively recovered during the study. Participants responded the Beck Depression Inventory-II and Brief Symptom Inventory. To determine the validity of the mean and standard deviation, item-total internal consistency and alpha coefficients were calculated for each item. Total reliability coefficient was obtained 0.913 [9]. To determine the validity of the questionnaire, factor analysis and construct validity were used. Cronbach's alpha coefficient for this questionnaire was 0.873 . Tashakori and Mehryarin 1994 obtained reliability coefficient of this questionnaire as 0.78 in Iran [10].

Edinburgh inventory was designed by Cox et al. in 1987 and includes symptoms of anxiety and concern. This tool is a widely used screening tool and there are different versions of this questionnaire in several languages [8]. The validity of Edinburgh inventory has been also investigated for screening postnatal depression with different scores, in several studies. Numerous studies have confirmed the validity of the instrument including studies by Jamil et al. (2006) with a sensitivity of $90 \%$ and a specificity of $60 \%$, and Tesfaye et al. (2010) with a sensitivity of $78.9 \%$ and a specificity of $75.3 \%$. In Iran, a 2010 study by Kamranpour et al. revealed the Cronbach's alpha more than 0.7, which confirms the reliability of the questionnaire [10]-[12].

\section{Findings}

According to the results of this study, in terms of age, there were no significant statistical differences between parents of term and preterm babies. However, higher levels of education in fathers of term infants in comparison to preterm infants, was obtained with Mann-Whitney test (Because of four classes for education including primary, pre high school, high school, university). This was a significant difference $(\mathrm{p}<0.001)$.

Most mothers of both term and preterm groups were housewives (Chi-Square test: 84.1\%). The majority of fathers of both term (Chi-Square test: 69.5\%) and preterm (Chi-Square test: 78\%) groups were self-employed. 
120 to 240 USD In $41.5 \%$ of term group, and 240 to 300 USD was the average monthly salary of $46.3 \%$ of preterm group. About housing, most of the participants (37.7\%) were owner of their place of residence and others lived in rental housing, governmental housing or lived with their families. Chi-Square test in two groups of parents showed no significant difference in terms of housing type, income and jobs (Table 1).

Most study units in both groups experienced their first childbirth. Chi-Square test in terms of number of childbirths, number of live births, number of sons and daughters, and gender of infant, type of labor, history of abortion, stillbirth's history, differences between the two groups were not significant.

Need for hospitalization ( $\mathrm{p}<0.001)$ and admission to neonatal intensive care unit $(\mathrm{p}<0.001)$ showed statistically significant difference between two groups of infants (Table 2).

According to the current study results, mothers in term-infant group (25.6\%) and mothers in preterm-infant group (24.4\%) suffered from postpartum depression and chi-square test result showed no significant difference between the two groups in this regard $(\mathrm{p}=0.85)$. Also, the majority of fathers in term group $(91.5 \%)$ and in preterm group (80.5\%) were not depressed. According to the Mann-Whitney test, fathers showed significant difference $(\mathrm{p}=0.045)$ in depression (Table 3).

Most fathers in the term group (78\%) and in the preterm group (63.4\%) claimed the pregnancies were planned. Chi-square test revealed a significant difference between the two groups in terms of planned or unintended pregnancy $(\mathrm{p}<0.001)$. Likewise, most mothers in the term group $(75.6 \%)$ and mothers in the preterm group (59.8\%) had planned pregnancies. Chi-square test revealed a significant difference between the two groups in terms of planned or unintended pregnancy $(p=0.004)$ (Table 4).

McNemar test results showed that term infants' parents $(\mathrm{p}=0.077, \mathrm{~K}=0.322)$, and preterm infants' parents $(\mathrm{p}=0.144, \mathrm{k}=0.17)$ agreed with each other on the unintended pregnancy.

Logistic regression model showed no significant relationship between mother's age, mother's occupation, father's occupation, mother's education level, economic status, the number of pregnancies, preterm infant, birth weight, gestational age, history of infant hospitalization, history of hospitalization in NICU, planned pregnancy according to mother, planned pregnancy according to father, planned infant's gender according to mother and planned infant's gender according to father and maternal postpartum depression (Table 5). But according to this model, a significant relationship was observed between housing status, mother's age at marriage, infant's gender and maternal postpartum depression (Table 5).

Logistic regression model showed no significant relationship between father's age, father's occupation, education level, economic status, preterm infant, birth weight, gestational age, history of infant hospitalization, history of hospitalization in NICU, planned pregnancy according to mother, planned pregnancy according to father, planned infant's gender according to mother and planned infant's gender according to father and paternal postpartum depression. But according to this model, a significant relationship was observed between housing status, mother's age at marriage, infant's gender and maternal postpartum depression (Table 6).

Table 1. Data collected from demographic characteristics of parents of preterm and term infants.

\begin{tabular}{lccc}
\multicolumn{1}{c}{ Demographic data } & Term & $\underline{\text { Preterm }}$ & Result \\
\hline Parental age: & mean (SD) & mean (SD) & \\
Mother & $28.22 \pm 4.54$ & $27.6 \pm 6.25$ & $\mathrm{p}=0.53$ (t-test) \\
Father & $32.83 \pm 4.83$ & $31.78 \pm 6.22$ & $\mathrm{p}=0.23$ (t-test) \\
Parental education:(High school) & $\mathbf{N} /(\%)$ & & \\
Mother & $42(51.2)$ & $36(43.9)$ & $\mathrm{p}=0.88$ (Mann-Whitney U) \\
Father & $24(29.3)$ & $54(65.9)$ & $\mathrm{p}<0.001$ (Mann-Whitney U) \\
Parental job: & $\mathbf{N} /(\%)$ & & $\mathrm{p}=1$ (Chi-Square test) \\
Mother(housewife) & $69(84.1)$ & $69(84.1)$ & $\mathrm{p}=0.21$ (Chi-Square test) \\
Father (self-employed) & $57(69.5)$ & $64(78)$ & $\mathrm{p}=0.056$ (Mann-Whitney U) \\
Family's income level (self-employed)/N/(\%) & $34(41.5)$ & $38(46.3)$ & $\mathrm{p}=0.46$ (t-test) \\
Mother's marriage mean age (SD) & $20.63 \pm 3.4$ & $20.23 \pm 3.6$ & \\
\hline
\end{tabular}


Table 2. Obstetric characteristics of parents of preterm and term infants and characteristics of newborns.

\begin{tabular}{lccc}
\hline \multicolumn{1}{c}{ Pregnancy information } & Term & $\frac{\text { Preterm }}{\mathbf{N} / \mathbf{( \% )}}$ & Result \\
\hline First pregnancy & $37(45.1)$ & $40(48.8)$ & $\mathrm{p}=0.71$ (Mann-Whitney U) \\
Live child & $39(47.6)$ & $43(52.4)$ & $\mathrm{p}=0.87$ (Mann-Whitney U) \\
Abortion & $15(81.7)$ & $22(62.8)$ & $\mathrm{p}=0.19$ (Chi-Square) \\
Stillbirth & $6(7.3)$ & $11(13.4)$ & $\mathrm{p}=0.2$ (Chi-Square) \\
Natural childbirth & $38(46.3)$ & $47(57.3)$ & $\mathrm{p}=0.11$ (Chi-Square) \\
Mother's point of view about wanting pregnancy & $64(78)$ & $51(61.2)$ & $\mathrm{p}=0.01$ (Chi-Square) \\
Father's point of view about wanting pregnancy & $64(78)$ & $47(57.3)$ & $\mathrm{p}=0.03$ (Chi-Square) \\
Failure of contraceptive methods & $10(12.2)$ & $23(28)$ & $\mathrm{p}=0.01$ (Chi-Square) \\
Baby sex (son) & $50(61)$ & $45(54.9)$ & $\mathrm{p}=0.42$ (Chi-Square) \\
hospitalization's need & $9(11)$ & $48(58.5)$ & $\mathrm{p}<0.001$ (Fisher-Exact test) \\
NICU hospitalization & $3(3.7)$ & $43(52.4)$ & $\mathrm{p}<0.001$ (Chi-Square) \\
paying costs ability & $80(97.6)$ & $70(85.4)$ & $\mathrm{p}=0.01$ (Fisher-Exact test) \\
\hline
\end{tabular}

Table 3. Data collected about frequency distribution (absolute and relative) of Depression in mothers of both groups.

\begin{tabular}{cccc}
\hline $\begin{array}{c}\text { Birth type } \\
\text { Mother's Depression }\end{array}$ & $\frac{\text { Term }}{\mathbf{N}(\%)}$ & $\frac{\text { Preterm }}{\mathbf{N}(\%)}$ & $\chi^{2}$ \\
\hline Yes & $21(25.6)$ & $20(24.4)$ & $\mathrm{p}=0 / 85$ \\
N/A & $61(74.4)$ & $62(75.6)$ & \\
\hline
\end{tabular}

Table 4. Data collected about frequency distribution (absolute and relative) of Depression in fathers of both groups.

\begin{tabular}{cccc}
\hline Birth type & $\frac{\text { Term }}{\mathbf{N}(\%)}$ & $\frac{\text { Preterm }}{\mathbf{N}(\%)}$ & $\chi^{2}$ \\
Father's Depression & $75(91.5)$ & $66(80.5)$ & $\mathrm{p}=0 / 045$ \\
Non depressed & $4(4.9)$ & $10(12.2)$ & \\
Mild depression & $3(3 / 7)$ & $5(6 / 1)$ & \\
Moderate depression & 0 & $1(1 / 2)$ & \\
Severe depression & & \\
\hline
\end{tabular}

\section{Discussion and Conclusion}

Mental disorders such as depression include a wide range of disorders that can have a profound impact on people lives and interactions. Therefore, early diagnosis of these disorders can lead to faster treatment and improved quality of life. Mental disorders have examined parents of preterm infants in few studies and in most of the studies, fathers' condition evaluation is neglected.

The results of the this study in terms of depression are not consistent with those of Gambina et al. in 2011, which was conducted to compare the levels of stress, anxiety, and depression in preterm and term infant's mothers. In their study, mothers of delayed preterm infants experienced more depression than term infants' mothers [13].

The results of this study in terms of maternal depression is consistent with the results of Gary et al. in 2012, in Australia ,which was conducted with the goal of determining the parental stress levels and mental health in very preterm infants' mothers compared to mothers of term infants. In their study, no differences were observed between mothers of term and preterm infants in the results of the Edinburgh Postpartum Depression Scale. The difference between the methodology used by Gary and ours is that, we issued the questionnaires to the participants two months after birth to assess parents' mental disorders, but they did so 4 months after delivery. Furthermore, Gary et al. examined only mothers' mental state and fathers' mental state was neglected. Participants 
Table 5. Depression of mothers in Logistic regression model.

\begin{tabular}{lcccc}
\hline & B coefficient & Expected B (OR) & p-value & CI B coefficient \\
\hline Mothers age & 0.615 & 1.849 & 0.046 & $1.1012-3.379$ \\
Mother's job & -1.279 & 0.276 & 0.278 & $0.028-2.782$ \\
father's job & 0.493 & 1.638 & 0.380 & $0.544-4 / 931$ \\
Mother's education & -0.406 & 0.666 & 0.096 & $0.413-1.075$ \\
Marriage age & -0.486 & 0.615 & 0.048 & $0.380-0.995$ \\
Economic statue & -0.228 & 0.796 & $0 / 419$ & $0.457-1.385$ \\
House statue & 1.469 & 4.347 & 0.016 & $1.310-14.430$ \\
Number of pregnancy & 0.069 & 1.072 & 0.781 & $0.659-1.743$ \\
prematurity & -0.323 & 0.724 & 0.538 & $0.259-2.024$ \\
Sex of baby & 1.122 & 3.071 & 0.023 & $1.168-8.075$ \\
Birth weight & 0.252 & 1.286 & 0.428 & $0.690-2.396$ \\
Gestational age & -0.104 & 0.901 & 0.836 & $0.337-2.413$ \\
Baby hospitalization & -1.041 & 0.353 & 0.158 & $0.83-1.496$ \\
NICU hospitalization & 1.865 & 6.455 & 0.053 & $0.978-42.598$ \\
Wanted pregnancy from mother's viewpoint & -0.150 & 0.861 & 0.787 & $0.290-2.554$ \\
Wanted pregnancy from father's viewpoint & 0.605 & 1.831 & 0.308 & $0.573-5.850$ \\
Wanted baby gender from mother's view & -0.327 & 0.721 & 0.633 & $0.188-2.763$ \\
Wanted baby gender from father's view & -0.479 & 0.536 & 0.620 & $0.136-2.817$ \\
\hline
\end{tabular}

Table 6. Depression of fathers in Logistic regression model.

\begin{tabular}{lcccc}
\hline & B coefficient & Expected B (OR) & p-value & CI B coefficient \\
\hline Father's age & 0.174 & 1.190 & 0.430 & $0.772-1.835$ \\
Father's job & -0.378 & 0.685 & 0.545 & $0.202-2.326$ \\
Father's education & -0.630 & 0.532 & 0.008 & $0.335-0.847$ \\
House statue & 0.302 & 1.353 & 0.596 & $0.443-4.132$ \\
prematurity & -0.964 & 0.381 & 0.372 & $0.046-3.164$ \\
Sex of baby & 0.709 & 2.032 & 0.136 & $0.799-5.167$ \\
Birth weight & 0.427 & 1.532 & 0.209 & $0.788-2.979$ \\
Gestational age & -0.87 & 0.917 & 0.863 & $0.343-2.450$ \\
Baby hospitalization & -0.14 & 0.986 & 0.988 & $0.158-6.171$ \\
NICU hospitalization & -1.332 & 0.264 & 0.210 & $0.033-2.123$ \\
Wanted pregnancy from father's viewpoint & 0.932 & 2.540 & 0.106 & $0.821-7.855$ \\
Wanted pregnancy from mother's viewpoint & 1.011 & 2.748 & 0.058 & $0.967-7.803$ \\
Wanted baby gender from father's viewpoint & -0.301 & 0.740 & 0.662 & $0.192-2.851$ \\
Wanted baby gender from mother's view & -0.103 & 0.903 & 0.883 & $0.229-3.558$ \\
\hline
\end{tabular}

in the study of Gary et al. included mothers of infants delivered at 24 - 30 weeks gestation and they have infants were hospitalized to the Neonatal Intensive Care Unit. In the present study, mothers of preterm infants have babies with gestational age of 32 - 36 weeks and only 52.4\% of them hospitalized in NICU [14].

Findings of the current research in terms of depression are not consistent with the research findings of Yurdakul et al. in 2009, which was conducted to assess symptoms of depression and anxiety and attachment of mother to baby in NICU. They concluded that the number of mothers with higher scores of depression in NICU 
was significantly more than mothers in the control group, but no difference was observed between anxiety and the type of mother's relationship and attachment of mother with infant. Yurdakul et al. have some differences with the present study in terms of mothers of infants admitted to Neonatal Intensive Care Units. A mother with a healthy term baby, participated as a control group. in the study Furthermore, STAI scale was used to evaluate anxiety and the research data were collected from mothers whose babies were in NICU [15].

To the best of our knowledge, no study was found that examined parents' agreement on unintended pregnancy and only a few studies investigated the relationship between unintended pregnancy and postpartum depression.

Al-Dallal et al. conducted a cross-sectional descriptive study in 2012 on 250 Bahraini Arab women who attended primary health care centers 8 weeks after delivery to screen their newborn. In this study, participants were randomly selected. The Arabic Edinburgh Postnatal Depression Scale was used for screening symptoms of postpartum depression. A researcher-made questionnaire was used to reflect demographic, maternal, newborn and psychological risk factors for postpartum depression. The significance level was considered $5 \%$. With regard to demographic data, no significant relationship was observed between depression score $\geq 12$, and mother's age at marriage, education level, parents' occupation, mother's marital status or the presence of another woman for their husbands. No significant relationship was seen between postpartum depressive symptoms, newborn gender, type of delivery, multiparity, prematurity, infant health status, history of neonatal death, separation from the infant, breastfeeding or breastfeeding problem. No significant relationship was observed between the score of postpartum depression and planned pregnancy, the time of previous pregnancy and pregnancy during contraceptive use [2].

Abdulbari Bener conducted a hospital-based study in 2013 and 1659 Arab women in the postpartum period participated in the study. The study was conducted in the form of face-to-face interviews by a researcher-made questionnaire containing questions about demographic and personal data, anthropometric measurements, medical history, and maternal characteristics. Depression, anxiety and stress were examined by Depression Anxiety Stress Scale (DASS-21). Depression and anxiety were significantly higher among preterm infants' mothers compared to term infants' mothers. Risk of depression in mothers of preterm infants that had low birth weight was two times more than term infants' mothers. While the risk of anxiety in mothers of preterm infants with low birth was 2.7 times more than term infants' mothers. Young mothers with low education level and less family income were more depressed and anxious after preterm birth than term infants' mothers. Psychological distress was higher in preterm infants' mothers [3].

Diababa et al. conducted a cohort study in 2013 in Ethiopia to investigate the relationship of unintended pregnancy during pregnancy, with social support and symptoms of depression. A total of 627 women participated in this study and the Edinburgh Postpartum Depression Scale was used to assess depressive symptoms during pregnancy. The relationship between independent variables and depressive symptoms during pregnancy was examined by multivariate logistic regression. Prevalence of depression symptoms during pregnancy was $19.9 \%$ and score of 13 was taken as cut of point. Women who reported their pregnancies as unintended were likely to experience depression symptoms almost twice more than women with planned pregnancy. Women who reported moderate levels of social support were less likely to report depressive symptoms [1].

\section{Acknowledgements}

This research is derived from the master thesis in the Shahid Beheshti Medical University. We appreciate the cooperation by honorable research deputy of university and all participants in this study.

\section{Authors' Contribution}

None declared.

\section{Financial Disclosure}

None declared.

\section{Funding Support}

We kindly express our gratitude to Research Deputy of Shahid Beheshti Medical University for providing the financial support. 


\section{References}

[1] Dibaba, Y., Fantahun, M. and Hindin, M.J. (2013) The Association of Unwanted Pregnancy and Social Support with Depressive Symptoms in Pregnancy: Evidence from Rural Southwestern Ethiopia. BMC Pregnancy and Childbirth, 13, 2-8.

[2] Al Dallal, F.H. and Grant, I.N. (2012) Postnatal Depression among Bahraini Women: Prevalence of Symptoms and Psychosocial Risk Factors. Eastern Mediterranean Health Journal, 18, 439-445.

[3] Bener, A. (2013) Psychological Distress among Postpartum Mothers of Preterm Infants and Associated Factors: A Neglected Public Health Problems. Revista Brasileira de Psiquiatria, 35, 231-236. http://dx.doi.org/10.1590/1516-4446-2012-0821

[4] Dolatian, M., Mirabzadeh, A., Forouzan, A.S., Sajjadi, H., Majd, H.A. and Moafi, F. (2013) Preterm Delivery and Psycho-Social Determinants of Health Based on World Health Organization Model in Iran: A Narrative Review. Global Journal of Health Science, 5, 52-64.

[5] Melnyk, B.M., Crean, H.F., Feinstein, N.F. and Fairbanks, E. (2007) Maternal Anxiety and Depression after a Premature Infant's Discharge from the Neonatal Intensive Care Unit: Explanatory Effects of the Creating Opportunities for Parent Empowerment Program. Nursing Research, 57, 383-394. http://dx.doi.org/10.1097/NNR.0b013e3181906f59

[6] Gray, P.H., Edwards, D.M., O’Callaghan, M.J. and Cuskelly, M. (2012) Parenting Stress in Mothers of Preterm Infants during Early Infancy. Early Human Development, 88, 45-49. http://dx.doi.org/10.1016/j.earlhumdev.2011.06.014

[7] Beck, A.T., Steer, R.A. and Garbin, M.G. (1988) Psychometric Properties of the Beck Depression Inventory: TwentyFive Years of Evaluation. Clinical Psychology Review, 8, 77-100. http://dx.doi.org/10.1016/0272-7358(88)90050-5

[8] Kheirabadi, G.R., Maracy Mohammad, R., Akbaripour, S. and Masaeli, N. (2012) Psychometric Properties and Diagnostic Accuracy of the Edinburgh Postnatal Depression Scale in a Sample of Iranian Women. Iranian Journal of Medical Sciences (IJMS), 37, 32-38.

[9] Dobson, K.S. and Mohammad Khani, P. (2007) Psychometric Characteristics Beck Depression Inventory-2 in a Large Sample of Patients with Major Depressive Disorder. Iranian Journal of Rehabilitation in Mental Disorders, 8, 80-86.

[10] Azkhosh, M. (2008) Use of Psychiatric Tests and Clinical Diagnosis. 3rd Edition, Ravan, Tehran, 224-226.

[11] Gemmill, A., Leigh, B., Ericksen, J. and Milgrom, J. (2006) A Survey of the Clinical Acceptability of Screening for Postnatal Depression in Depressed and Non-Depressed Women. BMC Public Health, 6, 1-8.

[12] Tesfaye, M., Hanlon, C., Wondimageqn, D. and Alem, A. (2010) Detecting Postnatal Common Mental Disorders in Addis Ababa, Ethiopia: Validation of the Edinburgh Postnatal Depression Scale and Kessler Scales. Journal of Affective Disorders, 122, 102-108. http://dx.doi.org/10.1016/j.jad.2009.06.020

[13] Kamranpour, S.B. and Shakiba, M. (2012) Cesarean Section and Post Partum Depression. Iranian Journal of Obstetrics, Gyneocology and Infertility, 15, 56-62.

[14] Gambina, I., Soldera, G., Benevento, B., Trivellato, P., Visentin, S., Cavallin, F., et al. (2011) Postpartum Psychosocial Distress and Late Preterm Delivery. Journal of Reproductive and Infant Psychology, 29, 472-479. http://dx.doi.org/10.1080/02646838.2011.653962

[15] Yurdakul, Z., Akman, I., Kuscu, M.K., Karabekiroglu, A., Yaylali, G., Demir, F., et al. (2009) Maternal Psychological Problems Associated with Neonatal Intensive Care Admission. International Journal of Pediatrics, 5, 35-39. 